\title{
Use of ammoniated rice straw and tree leaves containing tannin as scarcity feed to Bakri lambs
}

\author{
M.Y. Mohamed*, M.M. Eissa, A.M. Saber, E.A. El-Wakeel, M.M. \\ Anwar, H. Ghobashy, F.E. Saba, A.R. Khattab, A.A.S. Mahgoub
}

Animal Production Research Institute (APRI), Agricultural Research center (ARC), NC 12618,Dokki, Giza, Egypt.

* Corresponding author: dr.yassin2005@gmail.com

Journal of Livestock Scienc (ISSN online 2277-6214) 12:184-197

Received on19/4/21, Accepted on 5/6/21, Published on 21/6/21

doi.10.33259/JLivestSci.2021.184-197

\begin{abstract}
To cover a shortage of animal food in arid regions in Egypt, this investigation has been carried out to compare the consequences of using ammoniated rice straw (ARS) with different proportions of tree foliage leaves (Cassava, CA; Acacia saligna, AS; and Acacia neloitca, AN) on ruminal fermentation and sheep performance. The superior six formulations of pastoral lime mixtures from CA and Acacia with ARS were selected according to chemical compositions and IVDMD values from among 72 formulations, which were tested in vitro. Thirty five growing Barki male lambs four months old and weighed $20.16 \pm 0.11 \mathrm{~kg}$ were divided into seven groups $(5 \mathrm{each})$; 0 : $40 \%$ concentrate feed mixture $(\mathrm{CFM})+60 \%$ ARS and served as control, T1: $40 \% \mathrm{CFM}+30 \% \mathrm{ARS}+30 \% \mathrm{CA}, \mathrm{T} 2$ : $40 \% \mathrm{CFM}+30 \% \mathrm{ARS}+30 \% \mathrm{AS}, \mathrm{T} 3: 40 \% \mathrm{CFM}+30 \% \mathrm{ARS}+30 \% \mathrm{AN} ; \mathrm{T} 4: 40 \% \mathrm{CFM}+30 \% \mathrm{ARS}+15 \% \mathrm{CA}+$ 15\% AS, T5: 40\% CFM + 30\% ARS + 15\% CA + 15\% AN, and T6: 40\% CFM + 30\% ARS + 15\% AS + 15\% AN. Protein content was higher in CA $(22.22 \%)$ compared to AS (16.67\%) and AN (15.99\%). ADF and NDF content in CA was the lowest. T1 and T2 showed the lowest potential ruminal gas and methane production than T4 and T5. T4, T5, and T6 improved $(\mathrm{P}<0.05)$ the degradability of DM, OM, and NDF, while reduced condensed tannins $(\mathrm{CT})$ levels and increased ammonia-N, short-chain fatty acids, microbial protein, and bacterial count. No influence of various treatments on $\mathrm{pH}$, protozoa count, butyric, and propionic acid. T3 group showed an increase in lambs'performance compared with the other groups.It could be concluded that the use of feed mixtures of tanninrich foliage and ammoniated rice foddercan used as a partial substitute for ARS andmay help in cover the scarcity of forage resources and resolve the negative effects of CT and reduce the methane released.
\end{abstract}

Keywords: Foliage leaves; ammoniated rice straw; tannin; neutral detergent fiber; microbial protein; Barki lambs 


\section{Introduction}

The scarcity of forage resources is one of the fundamental constraints of improving animal production in Egypt's arid and semi-arid regions. The indigenous natural pastures are the main feed sources in the Egyptian deserts, broadly spread in many areas of Egypt of the Mediterranean Sea and the Red Sea and also in Chile Archipelago (Martinez et al., 2012 \& 2015). Halophytes make up a large part of the natural range, especially shrubs and perennials, which Almost 70 percent of the total coverage accounts f plant species (Sleimi and Abdelly, 2003). So, feeding halophytes and crop residue is a feasible solution to minimize the expected problems of feed shortage in such areas.

Under semi-arid area conditions, small ruminants fed on trees and shrubs such as Cassava and Acacia to solve the attendant problems of low productivity (Eissa et al., 2016). Cassava leaves have been found to have a high nutritional value which can effectively boost the nutrition of small ruminants when preserved as hay. The crude protein (CP) content of Cassava leaves is in the range of 22-29\% of dry matter (DM) (El Shaer, 2010). Furthermore, it has been proven that Cassava leaves are rich in amino acids, comparable to soybean meal. However, such trees and shrubs foliage are generally rich in anti-nutritional factors, particularly tannins (Makkar, 2003). Feeding a mixture of these fodder shrubs could minimize or overcome the problems of palatability and toxic effects (Anbarasu et al., 2001; Patra et al., 2002; Shaker et al., 2014).The conventional approach to fodder trees is to study and exploit "single" species. While the use of mixtures of species thus appears to be desirable, there is little scientific information on which to base practical recommendations. Recent results quantified the associative effects in vitro of mixtures of different species of Egyptian fodder trees, identifying significant interactions. These suggest that there is considerable potential to develop feeding systems based on strategic combinations that result in added nutritive value. They can be achieved by capitalizing on the interactive processes, such as: protecting dietary protein with natural tannins to increase the amount of nitrogen that by-passes the rumen; diluting the effects of deleterious compounds; inducing associative impacts that result in an increased voluntary intake, and inducing associative effects on digestibility between the components of the mixture. Appropriate mixtures of tree foliage can result in an overall improvement in nutritive value and contribute to making more efficient use of the natural diversity of trees and shrubs in the arid regions, and hence helping to sustain it(Mohamed et al., 2020).

The lack of information about feeding the mixture of Cassava and Acacia shrubs with ammoniated rice straw and their effect on animal performance in arid areas of Egypt was the investigation's motive. Therefore, this experiment was intended to verify whether the blending of foliage leaves with roughage can enhance these plants' use and affect the fermentation kinetics of in vitro gas production and Barki lamb performance.

\section{Material and methods}

This study conducted at the Animal Production Research Station Borg El-Arab. Which stretches along 525 $\mathrm{km}$ on the Mediterranean Sea, west of Alexandria city at latitudes 21 and 31 north and longitudes 25 and 35 East. Preparation of plants

Cassava (CA), Acacia salign a(AS), and Acacia neloitca (AN) using careful leaf harvesting within 30 minutes in the morning mowed $5 \mathrm{~cm}$ above ground level, dried, and then separated the leaves from the woody branches. Leaves and twigs were obtained. After CA, AS, and AN dried, they were cut into small pieces (5 mm) to avoid bunching.

\section{Experimental design}

This study has been carried out to compare the consequences of using treated ammoniated rice straw (ARS) with different proportions of tree foliage leaves (Cassava, CA; Acacia saligna, AS; and Acacia neloitca, AN or their mixture) on ruminal fermentation and productive performance of growing lambs. The superior six formulations of pastoral lime mixtures from Cassava and Acacia with ARS were selected according to chemical compositions and IVDMD values from among 72 formulations, which were tested in vitro. Thirty five growing Barki male lambs four months old and weighed $20.16 \pm 0.11 \mathrm{~kg}$ were divided into seven groups (5 each);included T0: $40 \%$ concentrate feed mixture $(\mathrm{CFM})+60 \%$ ARS and served as control, T1: $40 \% \mathrm{CFM}+30 \% \mathrm{ARS}+30 \% \mathrm{CA}, \mathrm{T} 2: 40 \% \mathrm{CFM}+30 \%$ ARS + 30\% AS, T3: 40\% CFM + 30\% ARS + 30\% AN; T4: 40\% CFM + 30\% ARS + 15\% CA + 15\% AS, T5: $40 \%$ $\mathrm{CFM}+30 \% \mathrm{ARS}+15 \% \mathrm{CA}+15 \% \mathrm{AN}$, and T6: $40 \% \mathrm{CFM}+30 \% \mathrm{ARS}+15 \%$ AS + 15\% AN. Animals were weighed biweekly for 21 weeks, the first three weeks was an adaptation period, and the other 18 weeks were a feeding period. Feeds were given at 07:00 and 18:00 $\mathrm{h}$ in two balanced meals according to NRC (2007). The level of the ingredients in the concentrate portion was adjusted to maintain iso-protein -iso-caloric experimental rations. The chemical composition of the ingredients and tested rations are presented in Table (1). 


\section{Digestibility and nitrogen balance trial}

Digestibility trial conducted using 21Barki rams, 2-3 years old, and weighted $43.00 \pm 1.85 \mathrm{~kg}$, holding individually in metabolic cages. They have been divided into the previous seven groups. This trial continued for 22 days (15 days as an adaptation period followed by seven days as a collection period). Feed amounts, residuals, fecal output, and urine volume were recorded daily.Nitrogen balance $(\mathrm{NB}, \mathrm{g} / \mathrm{h} / \mathrm{d})$ was mathematically determined by the following equation; $\mathrm{NB}=$ total $\mathrm{N}$ intake $-($ fecal $\mathrm{N}+$ urine $\mathrm{N})$.

Chemical composition of samples

Feedstuff rations and fecal samples were analyzed according to AOAC (2003). Neutral and acid detergent fiber (NDF \& ADF) according to Van Soest et al. (1991), condensed tannins (CT) according to Makkaret al. (1993), total flavonoid according to Zhishen et al. (1999), and free radical scavenging activity on $\alpha$, $\alpha$-diphenyl- $\beta$ picrylhydrazyl (DPPH; $\mathrm{C}_{18} \mathrm{H}_{12} \mathrm{~N}_{5} \mathrm{O}_{6}$ ) was determined according to Blois (1958). Siddhuraju and Becker (2003) defined the total phenolic content of leaves calculated by the FolinCiocalteu process. Rumen fluid short-chain fatty acids (SCFA's) are evaluated by gas chromatography (Isac et al., 1994). Ammonia-N before incubation, buffer mixture, and after incubation was estimated by the phenol-hypochlorite method according to Broderick and Kang (1980).

Determination of gas production $(G P)$

A modified version determined rumen GP and methane $\left(\mathrm{CH}_{4}\right)$ production in vitro for foliage, according to Navarro-Villa et al. (2013). Kinetic GP parameters are determined by matching GP (mL/g OM) output in nonlinear regression models as defined in the model presented by France et al. (2000). The ratio of genuinely degraded organic matter (mg) to gas volume (ml) at twenty-four hours of incubation was used as a microbial synthesis efficiency index (Blümmel et al.,1997).Using the Menke et al. (1979) equation, the metabolizable energy (ME) are determined as follows: $\mathrm{ME}(\mathrm{MJ} / \mathrm{kg} \mathrm{DM})=2.20+0.136 \mathrm{GP}+0.057 \mathrm{CP}$.

\section{Blood samples}

At the end of the experiment, blood samples were taken from each animal in all treatments. The blood samples were taken at three hours post morning feeding. A sample of $15 \mathrm{ml}$ of blood per animal waswithdrawn from the jugular vein and directly collected into clean dried glass culture tubes after adding EDTA. The blood plasma was obtained by centrifuging the blood samples soon after collection at $4000 \mathrm{rpm}$ for 15 minutes. Blood plasma was transferred into clean dried glass vials and then stored in a deep freezer at $-20^{\circ} \mathrm{C}$ for subsequent specific chemical analysis.

Economic efficiency and Statistical analysis

Based on local prices, economic efficiency was determined as total output/total input (where one-ton of CFM, CA, AS, ARS \& $\mathrm{kg} \mathrm{BW/lambs} \mathrm{were} \mathrm{2850,} \mathrm{550,} \mathrm{550,} 500$ \&45 L.E, respectively). Data were expressed as means $( \pm$ S.E.), and statistical analyses were performed with the General Linear Model (GLM) of SAS (2008). Duncan's New Multiple Range Test of the same SAS program was applied to determine significant differences among all tested treatments.

\section{Results and discussion}

The compositions of foliage leaves offered to Barki lambs are presented in Table (1). The crude protein (CP) was higher in CA (22.22\%) compared to AS (16.67\%) and AN (15.99\%), and ARS (3.09\%). The present results are consistent with the contents of the AS values reported by Shumuye and Yayneshet (2011). However, their values of CA tended to be smaller than those estimated by Oni et al. (2011), who reported that the CP content of CA ranged from 177 to $240 \mathrm{~g} / \mathrm{kg} \mathrm{DM}$, while AN was in the range from 14 to 26\% (Vimal et al., 1986).These salttolerant plants had higher crude protein $(\mathrm{CP})$, which could safely cover the essential nutrients requirements for animals.

Table (1) shows that the CP and EE were higher in CA, AS, and AN than their mixing as salt-tolerant plant mixture. These results agree with Shaker et al. (2014) and Helal et al. (2018), who said that the results showed that $\mathrm{CP}, \mathrm{EE}$, and ash contents were higher in alfalfa hay and berseem hay respectively than tree legumes mixture (Prosopis juliflora, Acacia saligna, Leucaena leucocephala), while this mixture had higher CF, NFE, and OM\%, These salt-tolerant plants had higher crude protein (CP), crude fiber (CF), nitrogen-free extract (NFE), and organic matter $(\mathrm{OM})$ which could safely cover the essential nutrients requirements for animals.

In the present study, all diets have adequate supplies of CP ranging from $8.13 \%$ (T0) to $13.87 \%$ (T1). These crud protein levels above the necessary microorganism's requirement $(7 \%)$ to encourage appropriate ruminal microflora growth and the host ruminant's maintenance requirement for CP (McDonald et al., 2002). These findings were agreed with Helal et al. (2018), who illustrated that salt-tolerant plants mixture was higher in CP (9.37\%) than 
Table 1. Chemical composition of the ingredients and tested rations (\% on DM basis).

\begin{tabular}{|l|l|l|l|l|l|l|l|l|}
\hline \multirow{2}{*}{ Items } & \multicolumn{6}{|c|}{ Chemical composition (\% on DM basis) } & \multirow{2}{*}{$\begin{array}{l}\text { CT } \\
\text { (g/kg DM) }\end{array}$} \\
\cline { 2 - 9 } & DM & OM & CP & EE & Ash & NDF & ADF & (kM \\
91.20 & 91.20 & 15.70 & 3.13 & 6.10 & 43.00 & 17.30 & nd \\
\hline ARS & 90.73 & 83.90 & 3.09 & 1.40 & 16.10 & 64.02 & 40.01 & nd \\
\hline CA & 88.76 & 90.36 & 22.22 & 4.24 & 9.64 & 49.14 & 33.28 & 20.58 \\
\hline AS & 88.65 & 90.65 & 16.67 & 2.95 & 9.35 & 55.23 & 35.58 & 25.03 \\
\hline AN & 90.02 & 90.82 & 15.99 & 3.66 & 9.82 & 52.55 & 34.77 & 15.61 \\
\hline T0 & 90.92 & 86.82 & 8.13 & 2.09 & 12.10 & 55.61 & 30.93 & nd \\
\hline T1 & 90.33 & 88.76 & 13.87 & 2.94 & 10.16 & 51.15 & 28.91 & 11.27 \\
\hline T2 & 90.29 & 88.85 & 12.21 & 2.56 & 10.08 & 52.98 & 29.60 & 12.31 \\
\hline T3 & 90.71 & 88.90 & 12.00 & 2.77 & 10.22 & 52.17 & 29.35 & 6.18 \\
\hline T4 & 90.31 & 88.80 & 13.04 & 2.75 & 10.12 & 51.25 & 29.25 & 11.79 \\
\hline T5 & 90.52 & 88.83 & 12.94 & 2.86 & 10.19 & 51.66 & 29.13 & 8.73 \\
\hline T6 & 90.50 & 88.87 & 12.11 & 2.66 & 10.15 & 52.57 & 29.48 & 9.25 \\
\hline
\end{tabular}

$\mathrm{CFM}=$ Concentrate feed mixture $\mathrm{ARS}=\mathrm{Ammoniated}$ rice straw $\mathrm{CA}=$ Cassava $; \mathrm{AS}=$ Acacia saligna $; \mathrm{AN}=$ Acacia neloitca $; \mathrm{nd}=\mathrm{not}$ detected $; \mathrm{DM}=\mathrm{Dry}$ matter; $\mathrm{OM}=$ Organic matter; $\mathrm{CP}=$ Crud protein; $\mathrm{EE}=$ Ether extract; $\mathrm{CF}=\mathrm{Crud}$ fiber; $\mathrm{NDF}=$ Neutral detergent fiber; $\mathrm{ADF}=$ acid detergent fiber; $\mathrm{CT}=$ condensed tannins $; \mathrm{T} 0=40 \% \mathrm{CFM}+60 \% \mathrm{ARS} ; \mathrm{T} 1=40 \% \mathrm{CFM}+30 \% \mathrm{ARS}+30 \% \mathrm{CA} ; \mathrm{T} 2=40 \% \mathrm{CFM}+30 \% \mathrm{ARS}+30 \% \mathrm{AS} ; \mathrm{T} 3=40 \% \mathrm{CFM}+$ $30 \% \mathrm{ARS}+30 \% \mathrm{AN} ; \mathrm{T} 4=40 \% \mathrm{CFM}+30 \% \mathrm{ARS}+15 \% \mathrm{CA}+15 \% \mathrm{AS} ; \mathrm{T} 5=40 \% \mathrm{CFM}+30 \% \mathrm{ARS}+15 \% \mathrm{CA}+15 \% \mathrm{AN} ; \mathrm{T} 6=40 \% \mathrm{CFM}+30 \% \mathrm{ARS}$ $+15 \%$ AS $+15 \%$ AN.

Table 2. Quantitative phytochemical analysis of the tested plants.

\begin{tabular}{|l|l|l|l|}
\hline Item & $\begin{array}{l}\text { Total Phenol } \\
\text { (GAE } \mathbf{~ m g / g ~ D M ) ~}\end{array}$ & $\begin{array}{l}\text { Total Flavonoid } \\
\text { (mg/g DM) }\end{array}$ & $\begin{array}{l}\text { Antioxidant activity } \\
\text { (\% of DPPH scavenging } \\
\text { activity })\end{array}$ \\
\hline CA & 99.73 & 12.22 & 65.68 \\
\hline $\mathbf{A S}$ & 108.60 & 14.97 & 48.21 \\
\hline $\mathbf{A N}$ & 121.37 & 16.30 & 72.84 \\
\hline $\mathbf{C A}+\mathbf{A S}$ & 110.37 & 14.38 & 57.32 \\
\hline $\mathbf{C A}+\mathbf{A N}$ & 120.27 & 15.71 & 68.82 \\
\hline $\mathbf{C A}+\mathbf{A S}+\mathbf{A N}$ & 105.50 & 14.72 & 62.09 \\
\hline \multicolumn{2}{|l}{ CA }
\end{tabular}

Table 3. Prediction of total gas, methane production and degradabilities of DM, OM and NDF\% and ME

\begin{tabular}{|c|c|c|c|c|c|c|c|c|}
\hline \multirow{2}{*}{ Item } & \multicolumn{7}{|c|}{ Treatments } & \multirow{2}{*}{$\pm \mathrm{SE}$} \\
\hline & T0 & T1 & T2 & T3 & T4 & T5 & T6 & \\
\hline P-GP (ml/g OM) & $99.77^{\mathrm{a}}$ & $75.56^{\mathrm{d}}$ & $72.32^{\mathrm{e}}$ & $84.75^{\mathrm{c}}$ & $92.38^{b}$ & $94.14^{b}$ & $96.38^{\mathrm{a}}$ & 4.018 \\
\hline GP-R (ml/h) & $0.078^{\mathrm{a}}$ & $0.039^{\mathrm{c}}$ & $0.034^{\mathrm{c}}$ & $0.063^{b}$ & $0.031^{\mathrm{c}}$ & $0.046^{\mathrm{b}}$ & $0.025^{\mathrm{d}}$ & 0.004 \\
\hline P-CH4 (ml/gOM) & $23.90^{\mathrm{a}}$ & $13.37^{\mathrm{d}}$ & $12.04^{\mathrm{e}}$ & $15.37^{\mathrm{C}}$ & $17.87^{\mathrm{b}}$ & $19.24^{b}$ & $21.93^{\mathrm{a}}$ & 1.275 \\
\hline CH4-R (ml/h) & $0.055^{\mathrm{a}}$ & $0.039^{\mathrm{c}}$ & $0.037^{\mathrm{c}}$ & $0.044^{\mathrm{b}}$ & $0.037^{\mathrm{c}}$ & $0.042^{b}$ & $0.018^{\mathrm{d}}$ & 0.004 \\
\hline P-CH4 / P-GP & $0.240^{\mathrm{a}}$ & $0.176^{\mathrm{d}}$ & $0.169^{\mathrm{e}}$ & $0.182^{c}$ & $0.193^{b}$ & $0.205^{b}$ & $0.228^{\mathrm{a}}$ & 0.016 \\
\hline IVDMD(\%) & $29.54^{\mathrm{a}}$ & $21.74^{\mathrm{d}}$ & $20.90^{\mathrm{e}}$ & $22.87^{\mathrm{d}}$ & $24.33^{\mathrm{c}}$ & $26.07^{b}$ & $25.09^{b}$ & 0.989 \\
\hline IVOMD(\%) & $28.26^{\mathrm{a}}$ & $24.06^{\mathrm{d}}$ & $23.96^{\mathrm{d}}$ & $24.92^{\mathrm{d}}$ & $25.21^{\mathrm{c}}$ & $27.05^{b}$ & $26.08^{b}$ & 0.533 \\
\hline IVNDFD(\%) & $29.76^{\mathrm{a}}$ & $22.56^{\mathrm{d}}$ & $21.35^{\mathrm{e}}$ & $23.62^{\mathrm{c}}$ & $24.77^{\mathrm{c}}$ & $25.36^{b}$ & $26.62^{b}$ & 1.271 \\
\hline ME (MJ/kg DM) & $5.41^{\mathrm{c}}$ & $9.71^{b}$ & $9.69^{b}$ & $11.97^{\mathrm{a}}$ & $10.26^{\mathrm{b}}$ & $11.44^{\mathrm{a}}$ & $9.41^{b}$ & 0.265 \\
\hline
\end{tabular}

$\mathrm{a}, \mathrm{b}, \mathrm{c}, \mathrm{d}$ and e Means in the same row with different superscript are significantly different $(P<0.05) . \mathrm{P}-\mathrm{GP}=\mathrm{Potential}$ gas production; GP-R= Gas production rate; $\mathrm{P}-\mathrm{CH} 4=$ Methane production efficiency; $\mathrm{CH} 4-\mathrm{R}=$ Methane production rate; $\mathrm{P}-\mathrm{CH} 4 / \mathrm{P}-\mathrm{GP}=$ methane production/gas production; IVDMD= In vitro dry matter degradability; IVOMD= In vitro organic matter degradability; IVNDFD= In vitro neutral detergent fiber degradibility; $\mathrm{ME}=$ Metabolizable energy. 
Table 4. Correlation between CT intakes vs. in-vitro NDF degradability, SCFA's, MP, GP, CH4 production for diets containing foliage leaves.

\begin{tabular}{|l|l|l|l|l|l|}
\hline Item & CT & IVNDFD & SCFA's & MP & CH4 \\
\hline IVNDFD & $0.019^{\text {ns }}$ & & & & \\
\hline SCFA's & $0.020^{\text {ns }}$ & $0.245^{\text {ns }}$ & & & \\
\hline MP & $0.070^{\text {ns }}$ & $0.655^{*}$ & $0.346^{\text {ns }}$ & & \\
\hline CH4 & $0.169^{\text {ns }}$ & $0.602^{*}$ & $0.591^{*}$ & $0.756^{*}$ & \\
\hline GP & $-0.202^{\text {ns }}$ & $0.479^{*}$ & $0.415^{\text {ns }}$ & $0.654^{*}$ & $0.648^{*}$ \\
\hline
\end{tabular}

$\mathrm{CT}=$ condensed tannins; IVNDFD=In vitro neutral detergent fiber degradability; SCFA's= Short chino fatty acids; MP=Microbial protein; $\mathrm{CH} 4=$ Methane production; GP= Gas production.

Table 5. Effect of tested diets on pH, NH3-N and SCFA's, MP and Bacteria count in vitro incubation (at 96 hrs) of lambs' rumen fluid.

\begin{tabular}{|l|l|l|l|l|l|l|l|l|}
\hline \multirow{2}{*}{ Item } & \multicolumn{9}{c|}{ Treatments } & \pm SE \\
\cline { 2 - 9 } & T0 & T1 & T2 & T3 & T4 & T5 & T6 & \\
\hline pH & 6.90 & 6.72 & 6.87 & 6.69 & 6.8 & 6.71 & 6.82 & 0.138 \\
\hline NH3-N $(\mathrm{mg} / \mathrm{dl})$ & $\mathbf{3 8 . 5 9 ^ { \mathrm { a } }}$ & $12.69^{\mathrm{d}}$ & $7.19^{\mathrm{e}}$ & $23.71^{\mathrm{c}}$ & $19.41 \mathrm{~b}^{\mathrm{c}}$ & $28.19^{\mathrm{b}}$ & $14.56^{\mathrm{d}}$ & 2.185 \\
\hline SCFA's $(\mathrm{meq} / \mathrm{dl})$ & $7.99^{\mathrm{b}}$ & $7.55 \mathrm{~b}^{\mathrm{b}}$ & $7.05^{\mathrm{c}}$ & $8.53^{\mathrm{a}}$ & $8.59^{\mathrm{a}}$ & $8.98^{\mathrm{a}}$ & $7.70^{\mathrm{b}}$ & 0.333 \\
\hline $\mathrm{MP}(\mathrm{mg} / \mathrm{dl})$ & $40.29^{\mathrm{b}}$ & $36.59^{\mathrm{c}}$ & $34.13^{\mathrm{d}}$ & $38.08^{\mathrm{c}}$ & $40.03^{\mathrm{b}}$ & $40.81^{\mathrm{b}}$ & $43.03^{\mathrm{a}}$ & 0.764 \\
\hline Bacteria count, $\left(\times 10^{5} / \mathrm{ml}\right)$ & $6.29^{\mathrm{c}}$ & $7.56^{\mathrm{b}}$ & $7.42^{\mathrm{b}}$ & $7.68^{\mathrm{b}}$ & $8.68^{\mathrm{a}}$ & $9.03^{\mathrm{a}}$ & $8.30^{\mathrm{a}}$ & 0.402 \\
\hline Protozoa count, $\left(\times 10^{3} / \mathrm{ml}\right)$ & 10.13 & 4.88 & 4.07 & 4.94 & 5.48 & 5.54 & 5.23 & 0.582 \\
\hline SCFA's fractionation $(\%)$ Acetic $(\mathrm{A})$ & $49.72^{\mathrm{d}}$ & $53.91^{\mathrm{c}}$ & $53.73^{\mathrm{c}}$ & $54.84^{\mathrm{c}}$ & $56.48^{\mathrm{b}}$ & $58.74^{\mathrm{a}}$ & $58.40^{\mathrm{a}}$ & 0.624 \\
\hline Propionic $(\mathrm{P})$ & 20.72 & 23.57 & 23.67 & 23.50 & 24.93 & 23.95 & 24.66 & 0.781 \\
\hline A/P & 2.45 & 2.29 & 2.27 & 2.33 & 2.28 & 2.46 & 2.37 & 0.076 \\
\hline Butyric & 9.32 & 11.52 & 11.44 & 11.71 & 12.06 & 12.09 & 12.10 & 0.212 \\
\hline
\end{tabular}

$\mathrm{a}, \mathrm{b}, \mathrm{c}, \mathrm{d}$ and e Means in the same row with different superscript are significantly different $(P<0.05)$. NH3-N= Ammonia nitrogen; SCFA' $\mathrm{s}=$ Short chain fatty acids; $\mathrm{MP}=$ Microbial protein.

Table 6. Mean nutrient intake, apparent digestibility coefficients, nutritive value and nitrogen balance of experimental ration offered to lambs.

\begin{tabular}{|c|c|c|c|c|c|c|c|c|}
\hline \multirow{2}{*}{ Item } & \multicolumn{7}{|c|}{ Treatments } & \multirow{2}{*}{$\pm \mathrm{SE}$} \\
\hline & T0 & T1 & $\mathbf{T 2}$ & T3 & T4 & T5 & T6 & \\
\hline \multicolumn{9}{|l|}{ Feed intake (FI) } \\
\hline Offer feed $(\mathrm{g} / \mathrm{h} / \mathrm{d})$ & 770 & 750 & 734 & 799 & 775 & 784.67 & 773.33 & 38.14 \\
\hline Refusal feed (g/h/d) & $338.90^{\mathrm{b}}$ & $250.90^{\mathrm{a}}$ & $132.93^{\mathrm{b}}$ & $121.17^{\mathrm{c}}$ & $108.73^{\mathrm{d}}$ & $107.07^{\mathrm{d}}$ & $96.10^{\mathrm{e}}$ & 5.56 \\
\hline Refusal feed \% & $44.01^{\mathrm{b}}$ & $33.45^{\mathrm{a}}$ & $18.11^{\mathrm{b}}$ & $15.27^{\mathrm{c}}$ & $14.03^{\mathrm{d}}$ & $13.65^{\mathrm{d}}$ & $12.43^{\mathrm{e}}$ & 0.814 \\
\hline Real FI (g/h/d) & 431.10 & 499.03 & 601.07 & 677.83 & 666.27 & 677.60 & 677.23 & 36.15 \\
\hline Real FI (\%) & $55.98^{\mathrm{d}}$ & $75.03^{\mathrm{e}}$ & $81.89^{\mathrm{d}}$ & $84.73^{\mathrm{c}}$ & $85.97^{\mathrm{b}}$ & $86.35^{\mathrm{b}}$ & $87.57^{\mathrm{a}}$ & 0.814 \\
\hline FI(g/kg weight) & 13.80 & 10.20 & 13.03 & 14.73 & 14.50 & 14.77 & 14.77 & 0.847 \\
\hline FI(g/kg weight $(0.075))$ & 35.93 & 23.93 & 33.97 & 38.37 & 37.77 & 38.43 & 38.37 & 2.167 \\
\hline CT intake $(\mathrm{g} / \mathrm{kg})$ & 0.00 & $11.27^{\mathrm{a}}$ & $12.31^{\mathrm{a}}$ & $6.18^{\mathrm{d}}$ & $11.79^{\mathrm{a}}$ & $8.73^{\mathrm{c}}$ & $9.25^{\mathrm{b}}$ & 0.737 \\
\hline \multicolumn{9}{|c|}{ Digestion coefficients (\%) } \\
\hline $\mathrm{DM}$ & $49.60^{\mathrm{e}}$ & $56.60^{b}$ & $55.38^{\mathrm{d}}$ & $59.20^{\mathrm{a}}$ & $56.02^{\mathrm{c}}$ & $57.57^{b}$ & $56.91^{b}$ & 0.706 \\
\hline $\mathrm{OM}$ & $43.85^{\mathrm{e}}$ & $58.85^{\mathrm{c}}$ & $57.31^{\mathrm{d}}$ & $61.31^{\mathrm{a}}$ & $58.13^{\mathrm{c}}$ & $59.24^{\mathrm{b}}$ & $58.50^{\mathrm{c}}$ & 0.838 \\
\hline$\overline{\mathrm{CP}}$ & 48.92 & 56.92 & 55.41 & 58.17 & 56.21 & 57.54 & 57.03 & 1.337 \\
\hline EE & $51.43^{\mathrm{d}}$ & $63.43^{\mathrm{c}}$ & $62.58^{\mathrm{c}}$ & $65.75^{\mathrm{b}}$ & $63.03^{\mathrm{c}}$ & $64.56^{\mathrm{b}}$ & $67.81^{\mathrm{a}}$ & 0.726 \\
\hline NDF & $47.84^{\mathrm{d}}$ & $55.84^{\mathrm{b}}$ & $53.76^{\mathrm{c}}$ & $57.03^{\mathrm{a}}$ & $53.23^{\mathrm{c}}$ & $55.91^{\mathrm{b}}$ & $53.59^{c}$ & 0.548 \\
\hline ADF & $48.12^{\mathrm{d}}$ & $51.12^{b}$ & $50.15^{\mathrm{c}}$ & $52.58^{\mathrm{a}}$ & $50.09^{c}$ & $51.81^{\mathrm{a}}$ & $51.17^{b}$ & 0.477 \\
\hline \multicolumn{9}{|c|}{ Nitrogen utilization $(\mathrm{g} / \mathrm{h} / \mathrm{d})$} \\
\hline $\mathrm{N}$ intake & $12.83^{\mathrm{a}}$ & $12.83^{\mathrm{a}}$ & $9.53^{c}$ & $10.90^{\mathrm{b}}$ & $12.00^{\mathrm{a}}$ & $12.33^{\mathrm{a}}$ & $12.27^{\mathrm{a}}$ & 0.620 \\
\hline N output & $12.30^{\mathrm{a}}$ & $12.30^{\mathrm{a}}$ & $9.22^{\mathrm{c}}$ & $10.12^{\mathrm{c}}$ & $11.55^{\mathrm{b}}$ & $11.76^{\mathrm{b}}$ & $11.64^{\mathrm{b}}$ & 0.622 \\
\hline $\mathrm{N}$ balance & $0.28^{\mathrm{d}}$ & $0.53^{\mathrm{c}}$ & $0.31^{\mathrm{d}}$ & $0.78^{\mathrm{a}}$ & $0.45^{\mathrm{c}}$ & $0.69^{\mathrm{b}}$ & $0.51^{\mathrm{c}}$ & 0.015 \\
\hline \multicolumn{9}{|l|}{ Nutritive value } \\
\hline TDN (\%) & $48.51^{\mathrm{c}}$ & $53.51^{\mathrm{b}}$ & $51.73^{\mathrm{d}}$ & $55.39^{\mathrm{a}}$ & $52.66^{\mathrm{d}}$ & $53.72^{\mathrm{b}}$ & $53.15^{\mathrm{b}}$ & 0.734 \\
\hline $\mathrm{DCP}(\%)$ & $4.21^{\mathrm{d}}$ & $7.21^{\mathrm{a}}$ & $5.47^{\mathrm{c}}$ & $5.85^{\mathrm{c}}$ & $6.33^{\mathrm{b}}$ & $6.54^{\mathrm{b}}$ & $6.45^{\mathrm{b}}$ & 0.155 \\
\hline
\end{tabular}

a, b,c,d and e Means in the same row with different superscript are significantly different $(P<0.05)$. DM= Dry matter; OM= Organic matter;

$\mathrm{CP}=$ Crud protein; $\mathrm{EE}=$ Ether extract; $\mathrm{CF}=\mathrm{Crud}$ fiber; $\mathrm{NDF}=$ Neutral detergent fiber; $\mathrm{ADF}=$ acid detergent fiber; $\mathrm{CT}=\mathrm{condensed}$ tannins; $\mathrm{TDN}=$

Total digestible nutrients; $\mathrm{DCP}=$ Digestible crude protein. 
the maintenance requirements for ruminants as recommended by Norton(2003), who determined that feeds contain less than $8 \% \mathrm{CP}$ could not provide the ammonia levels required by rumen microbes for optimum activity.

Furthermore, the ADF and NDF levels in CA were inferior to those of the other plants and their mixtures. These findings were agreed with Fayed et al. (2010), Shaker et al. (2014), and Helal et al. (2018) said that the mixing of plants affecting crude fiber (CF) values were decreased as increased portions of Atriplex. While, El-Waziry (2007) reported that mixing of fresh Acacia and Atriplex had increased the degradation of neutral detergent fiber (NDF), acid detergent fiber (ADF), acid detergent lignin (ADL), and hemicellulose.

The present results showed higher CT content in AS $(25.03 \mathrm{~g} / \mathrm{kg} \mathrm{DM})$ than CA $(20.58 \mathrm{~g} / \mathrm{kg} \mathrm{DM})$, while AN $(15.61 \mathrm{~g} / \mathrm{kg} \mathrm{DM})$ had the least content. The content of CT in AS was lower than this reported by Salem (2005) that was 113 and $63 \mathrm{~g} / \mathrm{kg} \mathrm{DM}$ in the winter and summer seasons, respectively, in comparison, Cassava's CT value ranges from 1.0 to $3.8 \mathrm{~g} / \mathrm{kg}$, estimated by Oni et al. (2011) while mixing of fresh shrubs indicated that tannins content was lower than three tolerant plants, these agree withEl-Waziry (2007),Fayed et al. (2010)andShaker et al. (2014) who reported that mixing of fresh shrubs indicated that tannins content was lower in the mixture than that of individual form. CT values are almost in the useful category ranged from 20 to $40 \mathrm{~g} / \mathrm{kg}$ DM confirmed by Nguyen et al. (2005).

Quantitative analysis of leaves' phytochemicals and their combinations are presented in Table (2). Total phenolic content ranged from 99.73 to $121.37 \mathrm{GAE} \mathrm{mg} / \mathrm{g} \mathrm{DM}$. Average flavonoids content varied from 12.22 to $16.30 \mathrm{mg} / \mathrm{g} \mathrm{DM}$, besides the concentration of antioxidant potential differed from 48.21 to $72.84 \%$. Ebanaet al. (1995), Alali et al. (2007), and Aryal et al.(2019) reported that total flavonoid content and phytochemicals have potent antimicrobial activity and free radical scavengers that avoid harm to oxidative cells, enhancing the productivity of animals.

As shown in Figures $(1 \& 2)$, in vitro cumulative $\mathrm{GP}$ and $\mathrm{CH}_{4}$ released for the experimental rations were roughly similar at various incubation times, except for T6, which showed more significant methane release. Potential-GP (P-GP) and Potential- $\mathrm{CH}_{4}\left(\mathrm{P}-\mathrm{CH}_{4}\right)$ from in vitro fermentation using an exponential equation of the various substrates of the experimental rations are shown in Table (3). Several studies have documented CT content's effects on methane reduction(Abo-Donia and Nagpal, 2015). Conversely, some studies found that tannins in sheep did not influence methanogenesis or even improved methane production (Śliwiński et al., 2002). In the current study, T1 and T2 were seen with the lowest P-GP and $\mathrm{P}_{-} \mathrm{CH}_{4}$ when utilized as fermentation substrates. The volume of total gas and methane released was increased by combining CA with AS or AN or their combinations with ARS. These findings are attributed to CT's presence, where the effect of ingested tannins on gas and methane production is shown in Figure (3). These findings are compatible with Hatew et al. (2016), who illustrated that raising CT values reduced peak rates of gas and methane production linearly and evaluated the digestibility of OM in vitro. The higher amount of GP found for diet supplemented AN leaves relative to AS or CA having relatively high CT levels (Table 3). Some scholars have pointed out many theories for how methanogenesis is prevented by CT; none of them have been confirmed. The $1^{\text {st }}$ assumption is that CT serves as a drain of hydrogen (Naumann et al., 2013a). The $2^{\text {nd }}$ theory is that CT specifically acts on rumen methanogens ( $\mathrm{Ng}$ et al., 2016). The $3^{\text {rd }}$ theory is that indirect prevention reduces the supply of nutrients to the rumen's microorganisms. Thereafter, the digestibility of the substrate decreases, which implicitly inhibits microbial communities of the rumen. Because CT binds to proteins (Saminathan et al., 2014), minerals (Lavin, 2012), lipids (Delehanty et al., 2007), or carbohydrates (Soares et al., 2012) also, CT binding to microbial enzymes can modulate their activity (Gonçalves et al., 2011).Naumann et al. (2013b) revealed a poor association between the CT-bound protein and decline in $\mathrm{CH}_{4}$.

In contrast to other therapies, T4, T5, and T6 demonstrated substantial $(\mathrm{P}<0.05)$ change in degradability of DM, OM, and NDF, The integration of ARS with AN improved $(\mathrm{P}<0.05)$ the benefit of ME relative to CA or AS. These findings, confirmed by Huang et al. (2010), revealed that lower pure CTs levels, 20-40 $\mathrm{mg} \mathrm{g}^{-1} \mathrm{DM}$, may decrease methane production without adverse effects on DM's degradability. Thus, there were negative correlations between IVDMD and the leaves' tannin content.Abo-DoniaandNagpal(2015)reported to the lowering of methane production is correlated with a decrease in DM degradability due to high CT concentrations. Consequently, tannins present in large amounts in foliage leaves in the NDF and ADF fractions and protein binding to the cell wall appear to be agents in digestibility reduction (Reed et al., 1990).

As seen in Table (4), there were positive correlations ( $\mathrm{r}=0.655 \&+0.602, \mathrm{P}<0.05)$ between both microbial protein (MP) \& $\mathrm{CH}_{4}$ and in vitro neutral detergent fiber degradability (IVNDFD). Also, between MP and both GP \& $\mathrm{CH}_{4}(\mathrm{r}=0.654 \& 0.756, \mathrm{P}<0.05)$, and between IVNDFD and GP $(\mathrm{r}=0.479, P<0.05)$. There have also been negative correlations noted between CT and GP $(\mathrm{r}=-0.202, P=0.422)$. Abo-Donia and Nagpal (2015) reported a linear lowering GP with rising CT concentrations. When including CT of L. leucocephala, GP was decreased up to $43 \%$. Likewise, methane production was reduced by 63\%. Besides, Hariadi and Santos (2010) reported that after 24 
Table 7. Effect of feeding experimental rations for Barki lambs on some blood serum parameters

\begin{tabular}{|c|c|c|c|c|c|c|c|c|}
\hline \multirow[t]{2}{*}{ Item } & \multicolumn{7}{|c|}{ Treatments } & \multirow[t]{2}{*}{$\pm \mathrm{SE}$} \\
\hline & T0 & T1 & $\mathbf{T 2}$ & T3 & T4 & T5 & T6 & \\
\hline TP (g/dl) & $5.22^{\mathrm{c}}$ & $6.75^{b}$ & $6.35^{b}$ & $8.65^{\mathrm{a}}$ & $7.16^{b}$ & $7.75^{\mathrm{a}}$ & $7.31^{\mathrm{a}}$ & \pm 0.40 \\
\hline ALB (g/dl) & $2.42^{\mathrm{c}}$ & $3.58^{\mathrm{b}}$ & $3.25^{b}$ & $4.82^{\mathrm{a}}$ & $3.78^{b}$ & $4.08^{\mathrm{a}}$ & $4.05^{\mathrm{a}}$ & \pm 0.26 \\
\hline GLO (g/dl) & 2.33 & 3.25 & 3.15 & 3.77 & 3.59 & 3.89 & 3.60 & \pm 0.19 \\
\hline ALB/GLO & $0.92^{c}$ & $1.14^{\mathrm{b}}$ & $1.09^{b}$ & $1.41^{\mathrm{a}}$ & $1.21^{b}$ & $1.65^{\mathrm{a}}$ & $1.35^{b}$ & $\pm \mathbf{0 . 0 7}$ \\
\hline Urea-N (mg/dl) & $30.10^{\mathrm{a}}$ & $24.10^{b}$ & $21.80^{\mathrm{c}}$ & $24.95^{\mathrm{b}}$ & $24.13^{b}$ & $24.15^{b}$ & $23.59^{b}$ & \pm 0.49 \\
\hline Creatinine (mg/dl) & $1.31^{b}$ & $1.82^{\mathrm{a}}$ & $1.82^{\mathrm{a}}$ & $1.43^{b}$ & $1.39^{b}$ & $1.89^{\mathrm{a}}$ & $1.98^{a}$ & \pm 0.12 \\
\hline AST (Ul) & 34.29 & 31.48 & 30.89 & 32.85 & 31.04 & 31.99 & 30.95 & \pm 0.50 \\
\hline ALT (Ul) & $19.80^{\mathrm{a}}$ & $17.20^{c}$ & $18.10^{b}$ & $18.62^{\mathrm{a}}$ & $17.96^{b}$ & $17.60^{b}$ & $17.51^{b}$ & \pm 0.38 \\
\hline
\end{tabular}

$\mathrm{a}, \mathrm{b}$ and $\mathrm{c}$ Means in the same row with different superscript are significantly different $(P<0.05) . \mathrm{TP}=$ Total protein; $\mathrm{ALB}=\mathrm{Albumin}$; $\mathrm{GLO}=$ Globulin; Urea-N= Urea nitrogen; $\mathrm{AST}=$ Aspartate aminotransferase; $\mathrm{ALT}=$ alanine aminotransferase

Table 8. Growth performance and economic efficiency of Barki lambs fed experimental rations.

\begin{tabular}{|l|l|l|l|l|l|l|l|l|}
\hline \multirow{2}{*}{ Item } & \multicolumn{7}{|c|}{ Treatments } & \pm SE \\
\cline { 2 - 9 } & T0 & T1 & T2 & T3 & T4 & T5 & T6 & \\
\hline No. of lambs & 5 & 5 & 5 & 5 & 5 & 5 & 5 & \\
\hline Feeding period, weeks & 18 & 18 & 18 & 18 & 18 & 18 & 18 & \\
\hline Initial weight, $(\mathrm{kg})$ & 20.12 & 20.28 & 20.32 & 20.08 & 20.02 & 20.32 & 20.02 & \pm 0.20 \\
\hline Final weight, (kg) & $36.74^{\mathrm{c}}$ & $40.68^{\mathrm{b}}$ & $37.73^{\mathrm{c}}$ & $42.09^{\mathrm{a}}$ & $39.62^{\mathrm{b}}$ & $41.39^{\mathrm{a}}$ & $39.90^{\mathrm{b}}$ & \pm 0.50 \\
\hline Total gain, (kg) & $16.62^{\mathrm{c}}$ & $20.40^{\mathrm{b}}$ & $17.41^{\mathrm{c}}$ & $22.01^{\mathrm{a}}$ & $19.60^{\mathrm{b}}$ & $21.07^{\mathrm{a}}$ & $19.88^{\mathrm{b}}$ & \pm 0.38 \\
\hline Daily body gain, (g) & $131.90^{\mathrm{d}}$ & $161.90^{\mathrm{b}}$ & $138.17^{\mathrm{d}}$ & $174.68^{\mathrm{a}}$ & $155.55^{\mathrm{c}}$ & $167.22^{\mathrm{b}}$ & $157.77^{\mathrm{c}}$ & \pm 0.003 \\
\hline Economic efficiency & & & & & & & & \\
\hline $\begin{array}{l}\text { Total feed coast(as } \\
\text { feed) /h/d (L.E.) }\end{array}$ & 1.30 & 1.33 & 1.28 & 1.34 & 1.31 & 1.32 & 1.23 & \\
\hline $\begin{array}{l}\text { Price of daily gain } \\
\text { (L.E.) }\end{array}$ & 5.10 & 4.50 & 4.59 & 5.13 & 4.65 & 4.89 & 3.93 & \\
\hline Efficiency (\%) & 3.20 & 3.47 & 3.67 & 3.92 & 3.64 & 3.80 & 3.28 & \\
\hline
\end{tabular}

$\mathrm{a}, \mathrm{b}$ and $\mathrm{c}$ Means in the same row with different superscript are significantly different $(P<0.05)$.

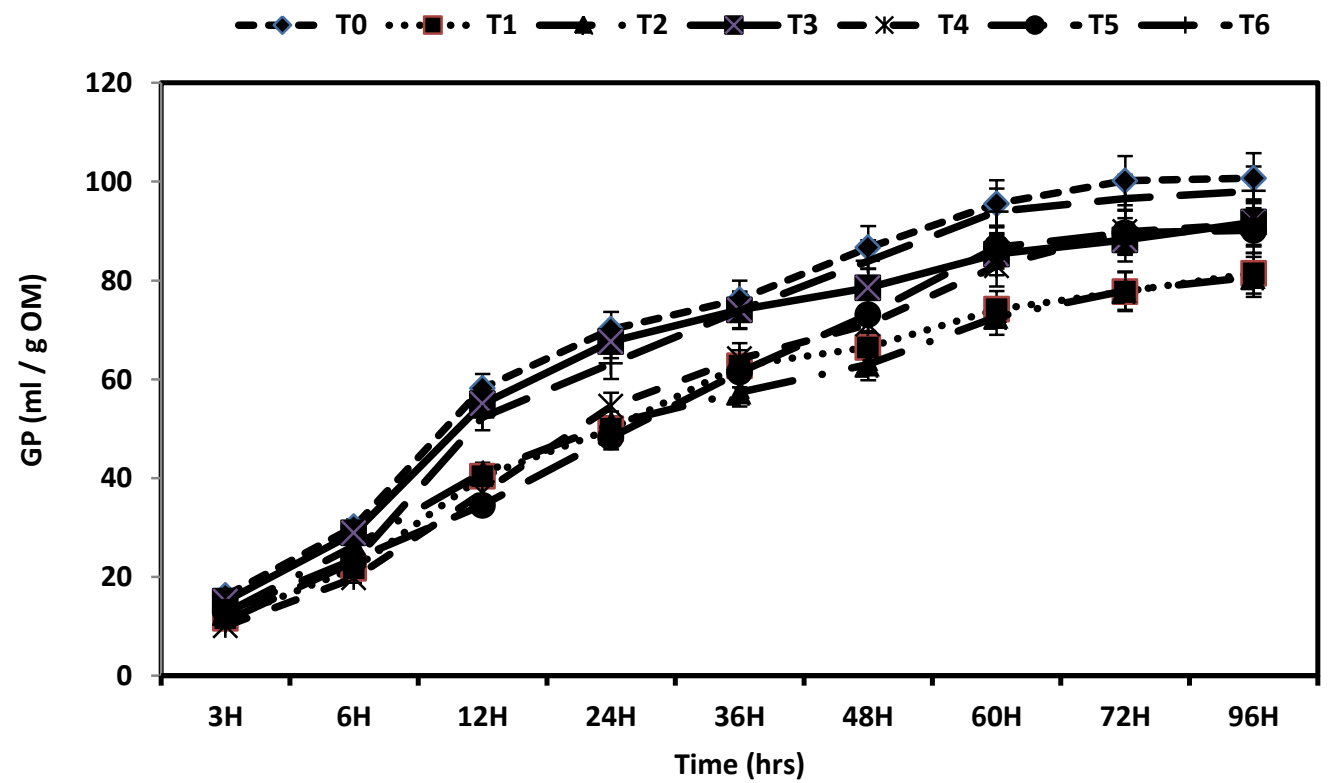

Fig. 1. Influence of using some foliage leaves on cumulative gas production (GP) at different incubation times in lambs'rumen fluid. 


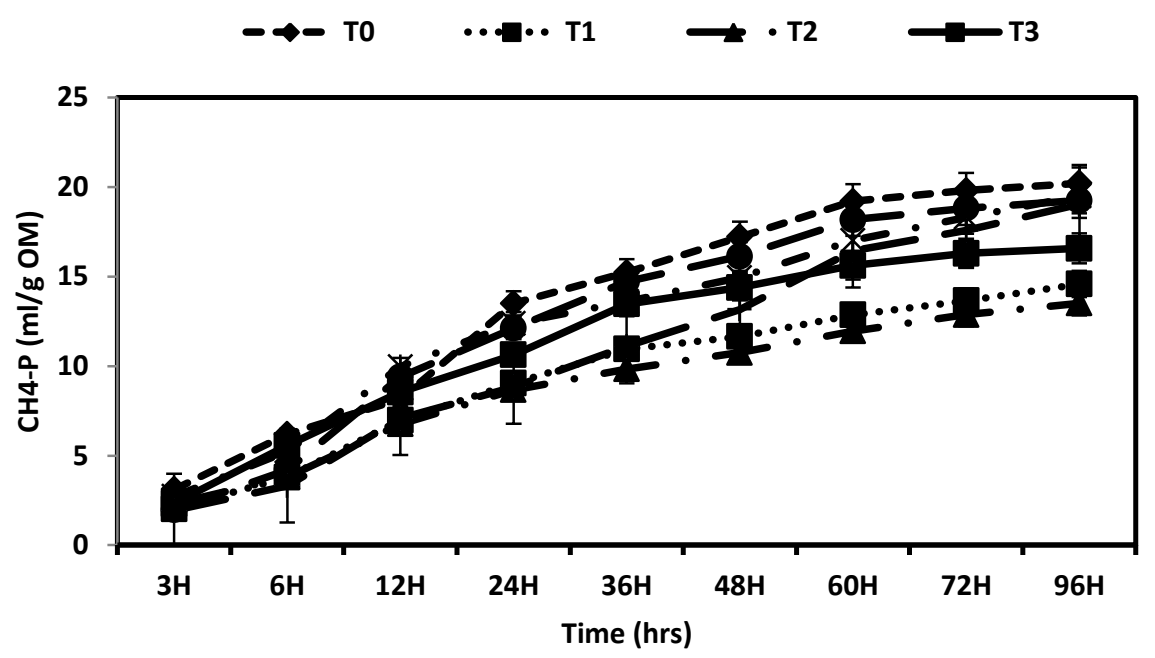

Fig. 2. Influence of using some foliage leaves on cumulative $\mathrm{CH}_{4}$ production at different incubation times in lambs' rumen fluid.

hours of incubation, a strong negative correlation $(\mathrm{r}=-0.60)$ between total tannins and in vitro $\mathrm{CH}_{4}$ production was recorded. The decrease in the overall production of methane may be attributed to the reduction in DM's degradability that might be related to the inclusion of CT-containing leaves in the tested diet mixtures provided to animals, as confirmed that by Gemeda and Hassen (2015).

Table (5) presents $\mathrm{pH}, \mathrm{NH} 3-\mathrm{N}$, SCFA's profile, microbial protein, and bacterial \&protozoa counts. The lower CT content has increased NH3-N, SCFA, MP, and bacteria levels, and vice versa. These findings were agreed with Naumann et al. (2017), who illustrated an inverse relationship between CT and SCFA's $(r=0.52)$. Tannins can improve the effectiveness of urea recycled into the rumen for ruminal NH3-N concentration. Hence, protein degradation rate and delamination in the rumen are reduced. In the current investigation, various treatments did no influence on $\mathrm{pH}$, protozoa count, propionic, and butyric count. Beaucheminet al.(2007) found that $20 \mathrm{~g} \mathrm{~kg}^{-1} \mathrm{DM}$ extract of quebracho tannin supplementation reduced acetic acid level and acetate/propionate ratio in the ruminant. The reduction in the acetate/propionate ratio due to the higher use of hydrogen to form propionate may be correlated with the inhibition of methane production by CT (Naumann et al., 2017). Gununet al.(2014) confirmed that the protozoan population was significantly reduced with CT supplementation in vitro. Bhatta et al.(2015) illustrated that the numbers of rumen ciliary protozoa reduced when the forage included CT of Azardirachta indica and Ficus bengalensis at levels of 13.8 and $26 \%$, respectively.

Data in Table (6) revealed that the use of foliage leaves separately with ARS raised significantly $(P<0.05)$ the level of feed refusal, particularly for T1 and T2 groups. Also, feed refusal decreased when various foliage was combined. Thus, feed intake reduced significantly $(P<0.05)$ when the foliage was separately introduced into the experimental rations. Waghorn et al. (1994) illustrated that ingestion of medium or low CT (<50 g kg-1 DM) plant species does not appear to affect feed intake. In contrast, it was substantially reduced by elevated CT content (> $50 \mathrm{~g}$ $\left.\mathrm{kg}^{-1} \mathrm{DM}\right)$. Also, sheep and camels are more resistant than other livestock to tannin-rich feed. Salem (2005) reported that utilizing sheep as models for livestock to characterize tanniniferous diets. In the current study, feed consumption and feed refused were due to the rising tannin content in some foliage leaves. The utilization of CT intake correlates to a similar feed refusal pattern, and the actual feed intake for the experimental diets is illustrated in Fig.(4).

The digestion experiments (Table 6) reported that the AN-containing diet was substantially superior to other diets. T1 and T3 increased the digestion coefficient of DM, OM, ADF, and NDF \% relative to T2. There were no significant variations among the experimental diets were found in protein digestion. This result could happen first because the CT significantly decreased the proteolytic enzyme's function and bacterial growth in sheep's rumen (Jones et al., 1993). Second, this could be attributed to condensed tannin-protein complexes produced at the rumen's $\mathrm{pH}$ level, which raises the obtainable protein known as ruminal bypass protein in the gastrointestinal tract (Min $e t$ $a l ., 2003)$. 
(a)

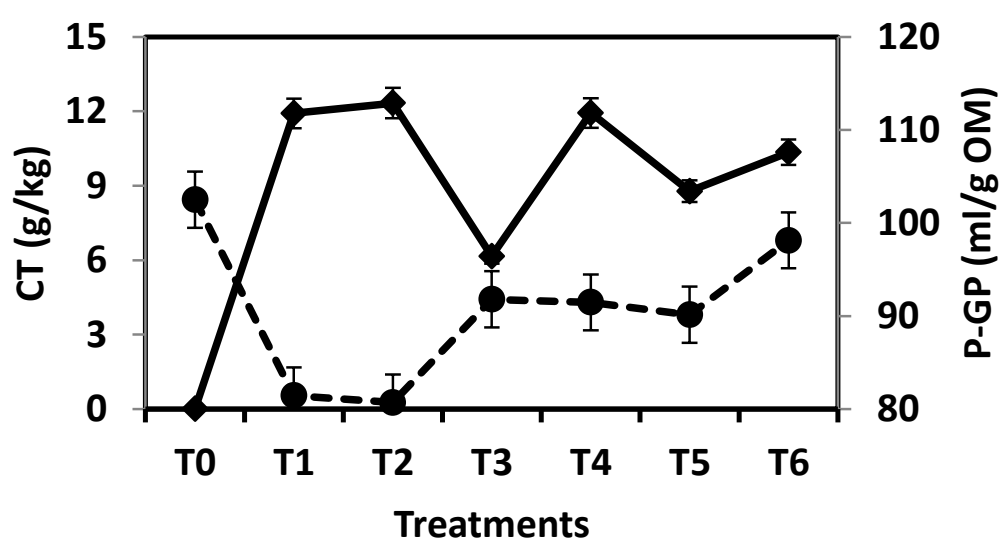

(b)

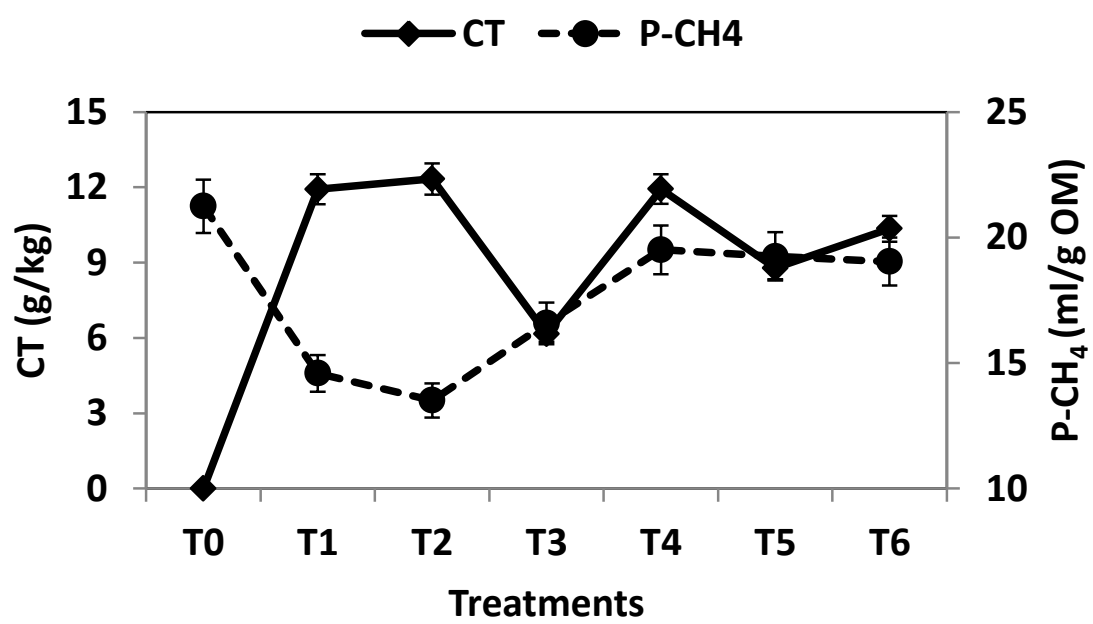

Fig. 3 Effect of condensed tannins (CT) consumption on (a) GP production and (b) $\mathrm{CH}_{4}$ emitted

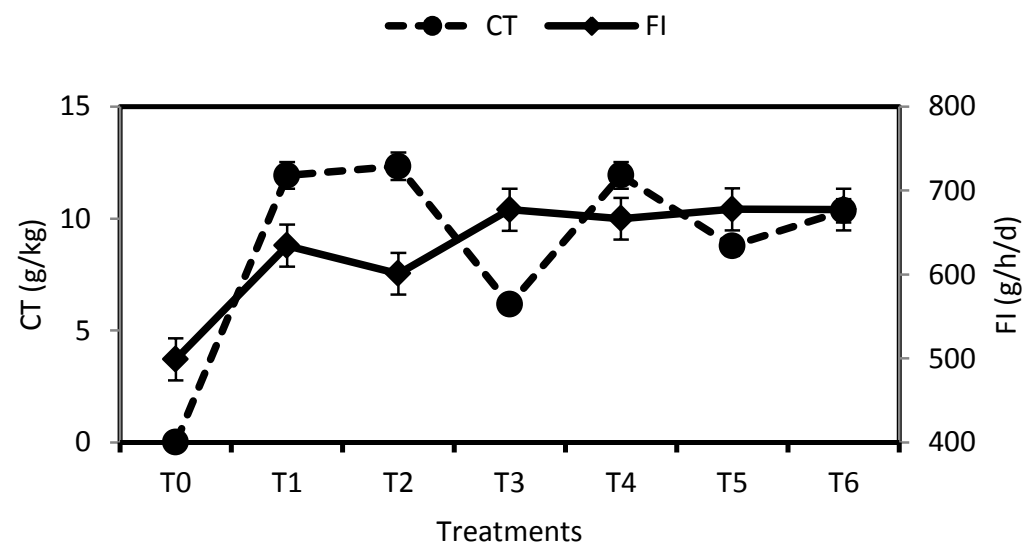

Fig. 4 Effect of condensed tannins (CT) consumption on feed intake (FI) 


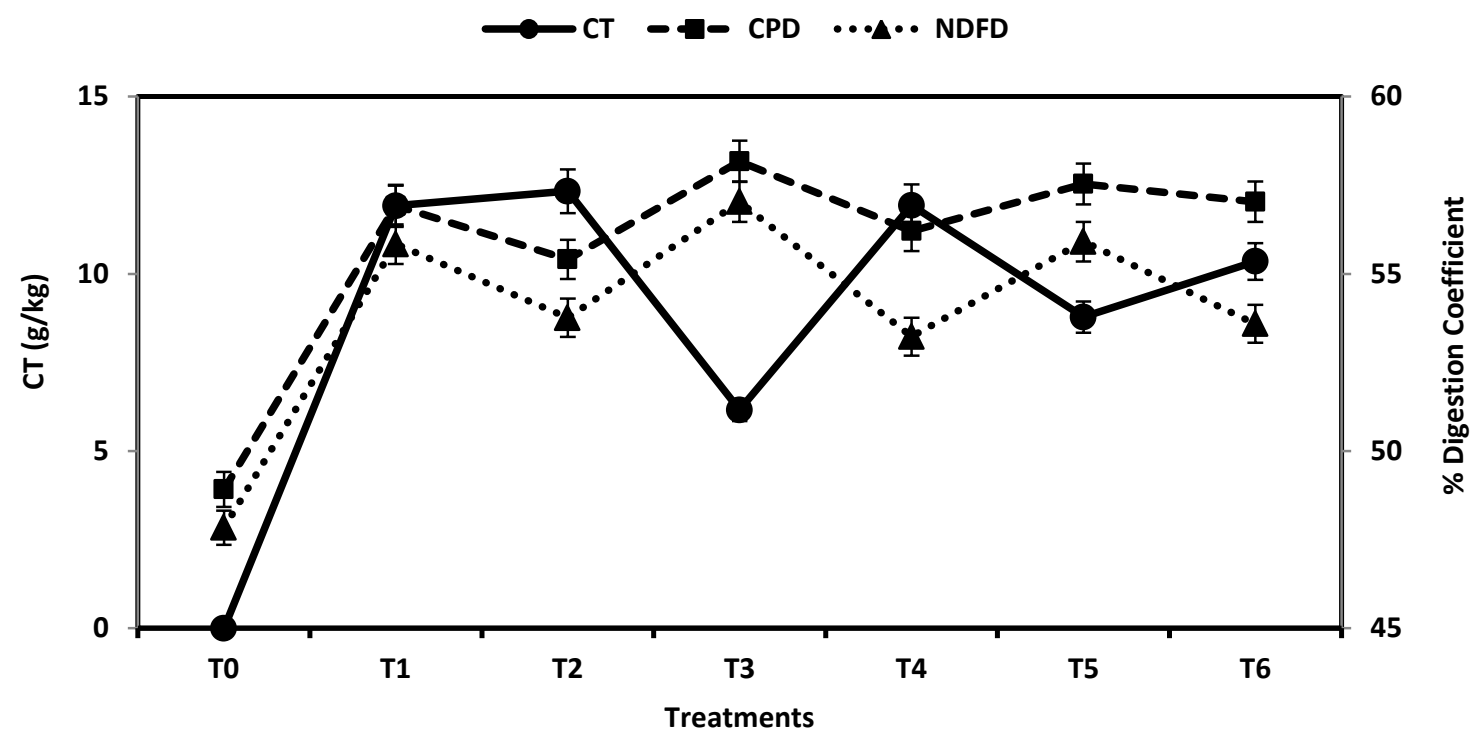

Fig. 5 Effect of condensedtannins(CT) consumption on crud protein (CP) and NDF digestibility

Higher tannin levels (> $50 \mathrm{~g} / \mathrm{kg} \mathrm{DM}$ ) can become an anti-nutritional factor in plant materials. They may decrease feed consumption and digestibility (Barry and McNabb, 1999) or milk production and growth rate (Waghorn et al., 1994). Therefore, tannins' useful influences in sheep are related to the flow and absorption of amino acids, especially for sheep fed on 2-4\% tannin in the diets (Min et al., 1999). Regarding fiber digestion, many researchers suggested that fiber degradation in the rumen can be significantly decreased in animals eating tanninrich diets (McSweeney et al., 2001). CT may reduce fiber digestibility by complicating with lignocelluloses, suppressing microbial digestibility (Auwal et al., 2014), or decreasing cellulolytic micro-organisms amount(Kavitha et al., 2013).

A feeding diet that includes CA or AN foliage leaves with ARS had a higher nitrogen intake than AS. The release of nitrogen follows the same food intake pattern, where nitrogen production was slightly lower $(P<0.05)$ with T2 (containing AS) compared to other treatments. N balance results found a significant $(P<0.05)$ rise in T3, $\mathrm{T} 4$, and T5 relative to control. These results are in agreement with Grainger et al. (2009). These findings might be attributed to foliage leaves' CT content, as presented in Fig.(5). The dissonance may interfere with the CT influences on nitrogen balance due to CT's source and molecular weight (Naumann et al.,2013b). TDN and DCP varied significantly $(P<0.05)$ among experimental groups. Feeding AN leaves mixed with ARS or supplemented with CA and AS may be greater than other experimental foliage.

The results provided in Table (7) showed that the blood parameters of treated lambs had minor variations. The lowest total protein (TP), albumin (ALB), globulin (GLO), and ALB/GLO ratio levels were observed with T2. These results are in agreement with Eissaet al. (2016), Shaker et al. (2014),and Sadek et al. (2020). This decline of TP in treated animals may be attributed to the plants' protein and tannins' content. Reed et al. (1990) found that high tannins in Acacia would likely reduce crude protein's digestibility. Mahmoud (2001) revealed that reducing GLO level in sheep could be attributed to a high tannins level that forms complexes with ration. So, T3 was greater than $\mathrm{T} 2$ in the blood protein profile. The same pattern was observed in serum urea-N. ALT and AST levels were greater with salt-tolerant shrubs mixture groups. The findings suggest that the measured blood parameters show minor variations because of shrubs source, where all values were within the standard ranges.

Feed efficiency and growth performance of Barki lambs fed experimental diets are showed in Table (8). The findings showed that a wide range of CT-dietary levels varied from 6.18 to $25.03 \mathrm{~g} / \mathrm{kg}$ DM (Table 1) that enhanced lambs' growth rate. There were significant differences $(P<0.05)$ among experimental groups in lambs' final weight, total body gain, and daily gain. T3 has the highest values $(42.09 \mathrm{~kg}, 22.01 \mathrm{~kg}, \& 174.68 \mathrm{~g}$, respectively), while T1 has the lowest values $(36.74 \mathrm{~kg}, 16.62 \mathrm{~kg}$, and $131.90 \mathrm{~g}$, respectively). These improvements could be attributed to the raised benefit of ME of treated diets compared to the ARS diet, which also enhanced the digestibility of DM, $\mathrm{OM}, \mathrm{ADF}$, and NDF\%. There is a negative association between the elevation of CT forages level (> $50 \mathrm{~g} \mathrm{CT} / \mathrm{kg}$ 
DM) and voluntary intake, palatability, digestion, and nitrogen retention in sheep. Concerning the feeding of ruminants, tannins are considered beneficial effects, such as increased protein intake, growth rate, wool and milk production, improved fertility, and animal welfare (Mueller-Harvey, 2006). This effect was attributed to the rise in proteins available post-rumen (Barry and McNabb, 1999; Eissa et al., 2015,Sadek et al., 2020).Shaker et al. (2014) said that such a salt-tolerant plant mixture's potentiality fulfills the animal requirements to maintain their body weight.

Economic efficiency (EE) represented in Table (8) indicated that feed cost $/ \mathrm{kg}$ body gain and economic efficiency improved with T3 (3.92\%) relative to other treatments. Eissa et al. (2015\& 2016) and Sadek et al. (2020) found that EE was significantly enhanced by substituting legume trees with ammoniated wheat straw in sheep diets.

\section{Conclusion}

It could be concluded that a mixture of treated ammoniated rice straw with different proportions of tree foliage leaves (Cassava, Acacia saligna, and Acacia neloitca or their mixture) could reduce the shortage of animal food in Egyptian arid region.They are a good indicator as feeding for sheep, help overcome CT's negative effect, decrease rumen-N absorption, reduce $\mathrm{CH} 4$ emission for livestock and increase the utilization of the available unpalatable salt-tolerant plants without any adverse effect on performance. So, we could use a mixture of treated ammoniated rice straw with tannin-rich foliage leaves as part of the animal diets better than ammoniated rice straw only. However, researchers still need to develop a series of searches from which recommendations can be developed.

\section{Animal Welfare Statement}

All research procedures were carried out in compliance with the standards set forth guidelines for the care and use of experimental animals by the Animal Ethics Committee of APRI, ARC, Egypt. Cannulated Barki sheep were well treated under good veterinary care. The cannulas have been installed by APRI ethical approval to the animals after sterilization and anaesthesia well with the provision of all appropriate conditions and remained under intensive veterinary care during the experimental period in Animal Production Research Station Borg El-Arab.All animals were kept under the same management and hygienic conditions. Lambs were kept in a ventilated barn and housed separately in shaded pens with concrete flooring and natural lighting through windows along either side of the building. The pens were cleaned in the morning and afternoon after feeding. Diets were provided at 8 am and $3.00 \mathrm{pm}$. and refusals were collected $24 \mathrm{~h}$ later. Fresh water and mineral licks are provided to animals as free choices.

\section{Declaration of interest}

All authors declare that there is no conflict of interest in this study.

\section{References}

1) Abo-Donia, F. M., \& Nagpal, A. K. (2015). In vitro evaluation of moth (Phaseolus aconitifolius) fodder, khejri (Prosopis cineraria) leaves and their combinations in camel. Indian Journal of Animal Nutrition, 32(2), 192-197.

2) Alali, F.Q., Tawaha, K., El-Elimat, T., Syouf, M., El-Fayad, M., Abulaila, K., Nielsen, S.J., Wheaton, W.D., Iii, J.O.F. and Oberlies, N.H., 2007. Antioxidant activity and total phenolic content of aqueous and methanolic extracts of Jordanian plants: an ICBG project. Natural Product Research, 21(12),1121-1131.

3) Anbarasu, C., Dutta, N., \& Sharma, K. (2001). Use of leafmeal mixture as a protein supplement in the rations of goats fed wheat straw. Animal Nutrition and Feed Technology, 1(2), 113-123.

4) AOAC (2003): Official methods of analysis (17 ${ }^{\text {th }}$ ed.) Association of official analytical chemists, Arlington, USA.

5) Aryal, S., Baniya, M. K., Danekhu, K., Kunwar, P., Gurung, R., \& Koirala, N. (2019). Total phenolic content, flavonoid content and antioxidant potential of wild vegetables from Western Nepal. Plants, 8(4), 96.

6) Auwal, M. S., Saka, S., Mairiga, I. A., Sanda, K. A., Shuaibu, A., \& Ibrahim, A. (2014). Preliminary phytochemical and elemental analysis of aqueous and fractionated pod extracts of Acacia nilotica (Thorn mimosa). In Veterinary research forum, 5(2), 95-100..

7) Barry, T. N., \& McNabb, W. C. (1999). The implications of condensed tannins on the nutritive value of temperate forages fed to ruminants. British Journal of Nutrition, 81(4), 263-272.

8) Beauchemin, K. A., McGinn, S. M., Martinez, T. F., \& McAllister, T. A. (2007). Use of condensed tannin extract from quebracho trees to reduce methane emissions from cattle. Journal of Animal Science, 85(8), 1990-1996.

9) Bhatta, R., Saravanan, M., Baruah, L., \& Prasad, C. S. (2015). Effects of graded levels of tannin-containing tropical tree leaves on in vitro rumen fermentation, total protozoa and methane production. Journal of Applied Microbiology, 118(3), 557564.

10) Blois, M. S. (1958). Antioxidant determinations by the use of a stable free radical. Nature, 181(4617), 1199-1200.

11) Blümmel, M., Steingaß, H., \& Becker, K. (1997). The relationship between in vitro gas production, in vitro microbial biomass yield and $15 \mathrm{~N}$ incorporation and its implications for the prediction of voluntary feed intake of roughages. British Journal of Nutrition, 77(6), 911-921. 
12) Broderick, G. A., \& Kang, J. H. (1980). Automated simultaneous determination of ammonia and total amino acids in ruminal fluid and in vitro media1. Journal of dairy science, 63(1), 64-75.

13) Delehanty, J. B., Johnson, B. J., Hickey, T. E., Pons, T., \&Ligler, F. S. (2007). Binding and neutralization of lipopolysaccharides by plant proanthocyanidins. Journal of natural products, 70(11), 1718-1724.

14) Ebana, R. U. B., Essien, A. I., \& Ekpa, O. D. (1995). Nutritional and potential medicinal values of the leaves Lasianthera Africana (BEAUV). Global journal of pure science, 1(1), 1-8.

15) Eissa, M. M., EL-Wakeel, E. L. A., Saber, A. M., Khattab, A. R., \&Sadek, W. M. A. (2015). Response of Barki lambs to diets containing Cassava and Treated wheat straw with Prosopis or Acacia Saligne (leaves \& twigs) under semi-arid area in Egypt. Egyptian J. Anim. Prod, 52(4), 79-87.

16) Eissa, M. M., Sadek, W. M. A., Khattab, A. R., \& Mohamed, H. G. (2016). Effects of feeding Cassava or Prosopis and their mixture along with ammoniated wheat straw on methane production (in vitro) and growth performance of growing Barki lambs under semi-arid condition. J. Animal and Poultry Prod., Mansoura Univ, 7(4), 129-139.

17) El Shaer, H. M. (2010). Halophytes and salt-tolerant plants as potential forage for ruminants in the Near East region. Small Ruminant Research, 91(1), 3-12.

18) El-Waziry, A. M. (2007). Nutritive value assessment of ensiling or mixing Acacia and Atriplex using in vitro gas production technique. Research journal of agriculture and biological sciences, 3(6), 605-614.

19) Fayed, A. M., El-Essawy, A. M., Eid, E. Y., Helal, H. G., Abdou, A. R., \& El Shaer, H. M. (2010). Utilization of alfalfa and atriplex for feeding sheep under saline conditions of south Sinai, Egypt. Journal of American Science, 6(1), 1447-1460.

20) France, J., Dijkstra, J., Dhanoa, M. S., Lopez, S., \&Bannink, A. (2000). Estimating the extent of degradation of ruminant feeds from a description of their gas production profiles observed in vitro: derivation of models and other mathematical considerations. British Journal of Nutrition, 83(2), 143-150.

21) Gemeda, B. S., \& Hassen, A. (2015). Effect oftannin and species variation on in vitro digestibility, gas, and methane production of tropical browse plants. Asian-Australasian Journal of Animal Sciences, 28(2), 188-199.

22) Gonçalves, R., Mateus, N., \& De Freitas, V. (2011). Inhibition of $\alpha$-amylase activity by condensed tannins. Food Chemistry, 125(2), 665-672.

23) Grainger, C., Clarke, T., Auldist, M. J., Beauchemin, K. A., McGinn, S. M., Waghorn, G. C., \& Eckard, R. J. (2009). Potential use of Acacia mearnsii condensed tannins to reduce methane emissions and nitrogen excretion from grazing dairy cows. Canadian Journal of Animal Science, 89(2), 241-251.

24) Gunun, P., Anantasook, N., Wanapat, M., Sirilaophaisan, S., Cherdthong, A., Wachirapakorn, C., \&Yuangklang, C. (2014). Effect of Mao (Antidesmathwaitesianum Mull. Arg.) seed supplementation on in vitro rumen protozoal population and digestibility using a gas production technique. KhonKaenAgr. J, 42(Suppl 4), 47-53.

25) Hariadi, B. T., \&Santoso, B. (2010). Evaluation of tropical plants containing tannin on in vitro methanogenesis and fermentation parameters using rumen fluid. Journal of the Science of Food and Agriculture, 90(3), 456-461.

26) Hatew, B., Stringano, E., Mueller-Harvey, I., Hendriks, W. H., Carbonero, C. H., Smith, L. M. J., \&Pellikaan, W. F. (2016). Impact of variation in structure of condensed tannins from sainfoin ( $\mathrm{O}$ nobrychisviciifolia) on in vitro ruminal methane production and fermentation characteristics. Journal of Animal Physiology and Animal Nutrition, 100(2), 348-360.

27) Helal, H. G., Nassar, M. S., Badawy, H. S., Eid, E. Y., \& El Shaer, H. M. (2018). Comparative nutritional studies of sheep and goats fed cultivated tree legumes mixture under desert condition. American-Eurasian Journal of Sustainable Agriculture, 12(1), 10-21.

32) Huang, X. D., Liang, J. B., Tan, H. Y., Yahya, R., Khamseekhiew, B., \& Ho, Y. W. (2010). Molecular weight and protein binding affinity of Leucaena condensed tannins and their effects on in vitro fermentation parameters. Animal Feed Science and Technology, 159(3-4), 81-87.

28) Isac, M. D., García, M. A., Aguilera, J. F., \& Alcaide, E. M. (1994). A comparative study of nutrient digestibility, kinetics of digestion and passage and rumen fermentation pattern in goats and sheep offered medium quality forages at the maintenance level of feeding. Archives of Animal Nutrition, 46(1), 37-50.

29) Jones, G. A., Jakober, K. D., Bae, H. D., McAllister, T. A., Cheng, K. J., \& Muir, A. D. (1993). Some interactions between condensed tannins of forage legumes, bovine serum albumin and five strains of proteolytic rumen bacteria. In Proceedings of the World Conference on Animal Production, Edmonton, Canada, 36, 68-69.

30) Kavitha, P. A., Kumar, P., Murthy, T. P. N., \& Gopinath, S. M. (2013). Methanolic extract of Acacia nilotica and antibacterial activity against Hospital isolates of Bengaluru district. Int. J. Latest Res. Sci. Technol, 2(1), 522-524.

31) Lavin, S. R. (2012). Plant phenolics and their potential role in mitigating iron overload disorder in wild animals. Journal of Zoo and Wildlife Medicine, 43(3s), S74-S82.

32) Mahmoud, H. A. (2001). Physiological and nutritional studies on sheep feeding certain halophytic plants in Sinai (Doctoral dissertation, M. Sc. Thesis, Fac. of Sci., Cairo University, Giza, Egypt).

33) Makkar, H. P. S. (2003). Effects and fate of tannins in ruminant animals, adaptation to tannins, and strategies to overcome detrimental effects of feeding tannin-rich feeds. Small ruminant research, 49(3), 241-256.

34) Makkar, H. P. S., Singh, B., Vats, S. K., \&Sood, R. P. (1993). Total phenols, tannins and condensed tannins in different parts of Rumex hastatus. Bioresource technology, 45(1), 69-71.

35) Martínez, M.E., Calderón, C., Uribe, H., de la Barra, R..(2012). Effect of management practices in the productive performance of three sheep breeds in the Chiloé Archipelago, Chile. Journal of Livestock Science 3, 57-66

36) Martínez, M.E., de la Barra, R., de la Fuente, F. (2015). Effect of early creep feeding in the performance of Chilota breed lambs. Journal of Livestock Science 6, 56-64 
37) McDonald, P., Edwards, R.A. \& Greenhalgh, J.F.D. (2002). Animal Nutrition. 6th Edition. Longman, London and New York. $543 \mathrm{p}$.

38) McSweeney, C. S., Palmer, B., McNeill, D. M., \& Krause, D. O. (2001). Microbial interactions with tannins: nutritional consequences for ruminants. Animal Feed Science and Technology, 91(1-2), 83-93.

39) Menke, K. H., Raab, L., Salewski, A., Steingass, H., Fritz, D., \& Schneider, W. (1979). The estimation of the digestibility and metabolizable energy content of ruminant feedingstuffs from the gas production when they are incubated with rumen liquor in vitro. The Journal of Agricultural Science, 93(1), 217-222.

40) Min, B. R., Barry, T. N., Attwood, G. T., \& McNabb, W. C. (2003). The effect of condensed tannins on the nutrition and health of ruminants fed fresh temperate forages: a review. Animal feed science and technology, 106(1-4), 3-19.

41) Min, B. R., McNabb, W. C., Barry, T. N., Kemp, P. D., Waghorn, G. C., \& McDonald, M. F. (1999). The effect of condensed tannins in Lotus corniculatus upon reproductive efficiency and wool production in sheep during late summer and autumn. The Journal of Agricultural Science, 132(3), 323-334.

42) Mohamed, Y. M., Saber, A. M., El-Wakeel, E. A., Anwar, M. M., Ghobashy, H., Abdel-Mawla, L. F., \&Eissa, M. M. (2020). Partial Replacement of Berseem Hay by Atriplex halimus and Atriplex nummularia Nutrients Utilization in Barki Sheep. International Journal of Current Microbiology and Applied Sciences, 9(7), 3169-3186.

43) Mueller-Harvey, I. (2006). Unravelling the conundrum of tannins in animal nutrition and health. Journal of the Science of Food and Agriculture, 86(13), 2010-2037.

44) Naumann, H. D., Muir, J. P., Lambert, B. D., Tedeschi, L. O., \&Kothmann, M. M. (2013a). Condensed tannins in the ruminant environment: a perspective on biological activity. Journal of Agricultural Sciences, 1(1), 8-20.

45) Naumann, H. D., Tedeschi, L. O., Hagerman, A. E., Lambert, B. D., \& Muir, J. P. (2013b). Methane emission and protein precipitating ability of condensed tannins from warm-season perennial legumes. In Energy and protein metabolism and nutrition in sustainable animal production (pp. 491-492). Wageningen Academic Publishers, Wageningen.

46) Naumann, H. D., Tedeschi, L. O., Zeller, W. E., \& Huntley, N. F. (2017). The role of condensed tannins in ruminant animal production: advances, limitations and future directions. RevistaBrasileira de Zootecnia, 46(12), 929-949.

47) Navarro-Villa, A., O'Brien, M., López, S., Boland, T. M., \&O'Kiely, P. (2013). In vitro rumen methane output of grasses and grass silages differing in fermentation characteristics using the gas-production technique (GPT). Grass and Forage Science, 68(2), 228-244.

48) Ng, F., Kittelmann, S., Patchett, M. L., Attwood, G. T., Janssen, P. H., Rakonjac, J., \&Gagic, D. (2016). An adhesin from hydrogen-utilizing rumen methanogen M ethanobrevibacterruminantium M 1 binds a broad range of hydrogen-producing microorganisms. Environmental microbiology, 18(9), 3010-3021.

49) Nguyen, T. M., Van Binh, D., \&Ørskov, E. R. (2005). Effect of foliages containing condensed tannins and on gastrointestinal parasites. Animal Feed Science and Technology, 121(1-2), 77-87.

50) Norton, B.W. (2003). The Nutritive value of tree legumes. In: Forage Tree Legume in Tropical Agriculture, Gutteridge R.C and Shelton H.M, (eds). pp.43

51) NRC, (2007). National Research Council, Committee on the Nutrient Requirements of Small Ruminants, Board on Agriculture, Division on Earth, \& Life Studies. Nutrient requirements of small ruminants: sheep, goats, cervids, and new world camelids.

52) Oni, A. O., Onwuka, C. F. I., Arigbede, O. M., Anele, U. Y., Oduguwa, O. O., Onifade, O. S., \& Tan, Z. L. (2011). Chemical composition and nutritive value of four varieties of cassava leaves grown in South-Western Nigeria. Journal of animal physiology and animal nutrition, 95(5), 583-590.

53) Patra, A. K., Sharma, K., Dutta, N., \&Pattanaik, A. K. (2002). Effect of partial replacement of dietary protein by a leaf meal mixture containing Leucaena leucocephala, Morus alba and Azadirachta indica on performance of goats. Asianaustralasian journal of animal sciences, 15(12), 1732-1737.

54) Reed, J. D., Soller, H., \& Woodward, A. (1990). Fodder tree and straw diets for sheep: intake, growth, digestibility and the effects of phenolics on nitrogen utilisation. Animal Feed Science and Technology, 30(1-2), 39-50.

55) Sadek, W. M. A., Elwakeel, E. A., Saber, A. M., Abdel-Mawla, L. F., Anwar, M. M., Ghobashy, H., \& Eissa, M. M. (2020). Comparative study of tanniniferous shrubs as an alternative source of feed on performance of sheep vs. goats under semidesert conditions of the north western-coast of Egypt Int. J. Curr. Res. Biosci. Plant Biol., 7(3), 1-14

56) Salem, A. F. Z. (2005). Impact of season of harvest on in vitro gas production and dry matter degradability of Acacia saligna leaves with inoculum from three ruminant species. Animal Feed Science and Technology, 123, 67-79.

57) Saminathan, M., Tan, H. Y., Sieo, C. C., Abdullah, N., Wong, C. M. V. L., Abdulmalek, E., \& Ho, Y. W. (2014). Polymerization degrees, molecular weights and protein-binding affinities of condensed tannin fractions from a Leucaena leucocephala hybrid. Molecules, 19(6), 7990-8010.

58) SAS Institute Inc. (2008). SAS/STAT Software, version 9.2. SAS

59) Shaker, Y.M., Ibrahim, N.H., Younis, F.E., \& El Shaer, H.M. (2014). Effect of feeding some salt tolerant fodder shrubs mixture on physiological performance of Shami goats in Southern Sinai, Egypt. Journal of American Science, 10(2s), 6677.

60) Shumuye, B., \&Yayneshet, T. (2011). Effect of feeding treated Acacia saligna (Labill.) HL Wendl. leaves on growth performance and digestibility in goats. J. Dryland Agric, 4(2), 341-347.

61) Siddhuraju, P., \& Becker, K. (2003). Studies on antioxidant activities of mucuna seed (Mucuna pruriens var utilis) extract and various non-protein amino/imino acids through in vitro models. Journal of the Science of Food and Agriculture, 83(14), 1517-1524. 
62) Sleimi, N., \& Abdelly, C. (2003). Protein of halophytes as animal fodder in Egypt. Chapter from book Salt-tolerance strategy of two halophyte species: Spartina alterniflora and Suaeda fruticosa. In Cash Crop Halophytes: Recent Studies (pp. 111-119). Springer, Dordrecht.

63) Śliwiński, B.J., Kreuzer, M., Wettstein, H.R., \&Machmüller, A. (2002). Rumen fermentation and nitrogen balance of lambs fed diets containing plant extracts rich in tannins and saponins, and associated emissions of nitrogen and methane. Archives of Animal Nutrition, 56(6), 379-392.

64) Soares, S., Mateus, N., \& de Freitas, V. (2012). Carbohydrates inhibit salivary proteins precipitation by condensed tannins. Journal of agricultural and food chemistry, 60(15), 3966-3972.

65) Van Soest, P.V., Robertson, J.B., \& Lewis, B.A. (1991). Methods for dietary fiber, neutral detergent fiber, and nonstarch polysaccharides in relation to animal nutrition. Journal of dairy science, 74(10), 3583-3597.

66) Vimal, O.P., \& Tyagi, P.D. (1986). Prosopis juliflora: chemistry and utilization. Role of Prosopis in Wasteland Development, 8.

67) Waghorn, G.C., Shelton, I.D. \& McNabb, W.C. (1994). Effect of condensed tannins in Lotus pedunculatas on its nutritive value for sheep. 1. Non-nitrogenous aspects. Journal of Agricultural Science, 123, 99-107.

68) Zhishen, J., Mengcheng, T., \& Jianming, W. (1999). The determination of flavonoid contents in mulberry and their scavenging effects on superoxide radicals. Food Chemistry, 64(4), 555-559. 